\title{
Benchmarking density functional theory methods for modelling cationic metal-argon complexes
}

\author{
Laia Delgado-Callico ${ }^{1, *}$, Piero Ferrari ${ }^{2, *, \dagger}$, Joost M. Bakker ${ }^{3}$, Francesca Baletto ${ }^{1}$ and Ewald Janssens ${ }^{2, \dagger}$ \\ ${ }^{1}$ Department of Physics, King's College London, London, UK \\ ${ }^{2}$ Quantum Solid-State Physics, Department of Physics and Astronomy, KU Leuven, Leuven, Belgium \\ ${ }^{3}$ Institute for Molecules and Materials, FELIX Laboratory, Radboud University, Nijmegen, The Netherlands \\ * These authors contributed equally to this work. \\ †ewald.janssens@kuleuven.be; piero.ferrari@kuleuve.be
}

\begin{abstract}
Noble gas chemistry is fascinating because noble gases can make formal chemical bonds with metal ions, despite their closed electronic configuration. Argon-metal ion complexes are particularly interesting since their bonding is halfway between dispersion and covalent interactions. Although many metal ion-noble gas complexes have been synthesized, there are still disagreeing theoretical descriptions about their bonding, which is not yet fully understood. Accurate experimental data is important as solid reference for theoretical methodologies, but such data is currently scarce for complexes of metal ion with noble gas atoms. We measured infrared spectra of $\operatorname{MAr}_{n}^{+}(n=3-5 ; M=\mathrm{Au}, \mathrm{Ag}, \mathrm{Pd})$ complexes and use these spectra as benchmark data for different theory levels within the density functional theory formalism. Several basis sets, exchange-correlation functionals, and the inclusion of dispersion corrections were considered. The agreement between the measured spectra and the calculations strongly depends on the applied level of theory. Functionals of a higher level of complexity do not consistently provide a better agreement with the experiment; this is particularly the case for the B3LYP hybrid functional that performs worse than the PBE GGA functional. On the other hand, the inclusion of dispersion corrections and the use of a large basis sets are crucial for a good description of the interaction between $M^{+}$and $\mathrm{Ar}_{\mathrm{n}}$.
\end{abstract}

\section{Introduction}

The chemistry of noble gases has a rich history [1,2]. While noble gases have long been considered inert due to their closed electronic configuration, in the last forty years researchers have shown they can form strong bonds with some metal ions. Already in 1977 , the $\mathrm{AuXe}^{+}$complex was observed by mass spectrometry [3], triggering theoretical studies to understand the interaction between a noble gas atom and a noble metal ion with closed electronic configuration such as $\mathrm{Au}^{+}$or $\mathrm{Ag}^{+}$.

In pioneering work, Pyykkö used MP2 and $\operatorname{CCSD}(\mathrm{T})$ computations to investigate $\mathrm{AuNg}^{+}(\mathrm{Ng}=\mathrm{He}, \mathrm{Ne}, \mathrm{Ar}, \mathrm{Kr}$ and $\mathrm{Xe}$ ) dimers [4], predicting large dissociation energies for dimers with $\mathrm{Ng}=\mathrm{Kr}$ and $\mathrm{Xe}(\sim 0.5-1 \mathrm{eV})$ and small dissociation energies for $\mathrm{Ng}=\mathrm{He}$ and $\mathrm{Ne}(\sim 0.02-0.05 \mathrm{eV})$. The dissociation energy of $\mathrm{AuAr}^{+}$lies in between $(\sim 0.2 \mathrm{eV})$. These results led to the conjecture that $\mathrm{He}$ and $\mathrm{Ne}$ interact weakly with $\mathrm{Au}^{+}$via the dispersion interaction, whereas the bonds of $\mathrm{Au}^{+}$with $\mathrm{Ar}, \mathrm{Kr}$ and $\mathrm{Xe}$ have a considerable covalent character. This idea was later disputed, by suggesting that the $\mathrm{Au}^{+}-\mathrm{Ng}$ bond can be described simply by long-range polarization and dispersion interactions [5].
Further theoretical work analyzing the electron density, however, supported the former interpretation [6, 7]. Nowadays, the consensus is that $\mathrm{Kr}$ and $\mathrm{Xe}$ bind notably covalently to $\mathrm{Au}^{+}$, while the $\mathrm{Au}^{+}-\mathrm{Ar}$ interaction is in between induced dipole-dipole and covalent [1].

Since then, a long list of metal-rare gas complexes of different charge state have been investigated, including $\mathrm{CuNg}_{n}{ }^{+}(n=1,2 ; \mathrm{Ng}=\mathrm{He}, \mathrm{Ar}, \mathrm{Kr})[8], \mathrm{MXe}(\mathrm{M}=\mathrm{Ni}, \mathrm{Pd}$, Pt) [9], $\mathrm{PdNg}_{2}$ and $\mathrm{PtNg}_{2}(\mathrm{Ng}=\mathrm{Ar}, \mathrm{Kr}, \mathrm{Xe})$ [10], $\mathrm{AgFKr}$ [11], and $\operatorname{AuAr}_{n}{ }^{+}(n=1-6)$ [12]. These complexes show a broad range of element-dependent interaction strengths. The comparison of $\mathrm{Au}$ with $\mathrm{Cu}$ and $\mathrm{Ag}$, which are all noble metal atoms, is particularly interesting. Studies have shown the ability of Au to form stronger bonds with noble gases, due to the prominent relativistic effects in the heavy Au atom. These effects provide the gold atom with a larger electron affinity [13], enhancing the electron charge transfer from the noble gas atom to $\mathrm{Au}[14,15]$. Recently, Echt and collaborators found "magic" complexes, i.e. species with higher intensities, in mass spectra of larger $\mathrm{AuNg}_{n}{ }^{+}(n \leq 15)$ and $\mathrm{AgNg}_{n}{ }^{+}(n \leq 25)$ complexes [16, 17]. The intensity pattern of $\mathrm{AuNg}_{n}{ }^{+}$is very distinct from the one of $\mathrm{AgNg}_{n}{ }^{+}$. 
A pronounced abundance maximum was found for $\mathrm{AuNg}_{2}{ }^{+}$, but not for the Ag cases. The authors speculated that this is a consequence of the higher covalent character in the interaction between $\mathrm{Au}^{+}$and noble gases.

Beyond single atoms, studies have focused on the interaction of small clusters with noble gases [18]. For example, Fielicke and co-workers combined infrared spectroscopy with DFT calculations to investigate the interaction of bimetallic $\mathrm{Au}_{n} \mathrm{Ag}_{m}{ }^{+}(n+m=3-5)$ clusters with $\operatorname{Ar}[19,20]$, showing that the bonding strength of the clusters towards Ar decreases with Ag content. Using the same approach, $\mathrm{Au}_{n}{ }^{+}(n \leq 9)$ [21], $\mathrm{Ag}_{n}{ }^{+}(n \leq 13)$ [22] and $\mathrm{Cu}_{n}{ }^{+}(n$ =3-10) [23] clusters have been studied with Ar as tagging element to address the structures of the metal clusters. For this type of studies, the cluster-Ar interaction is ideally weak and has no effect on the geometry of the metallic cluster, although this is not always the case. Neutral clusters, such as $\mathrm{Au}_{3} \mathrm{Kr}_{m}(m=1,2)$ [24] and $\mathrm{Au}_{n} \mathrm{Kr}(n \leq 15)$ [25, 26, 27], have also been investigated by infrared spectroscopy.

One needs to select an appropriate theory level to describe the metal/ion - noble gas complexes. In studies using infrared spectroscopy as the experimental tool, it is frequent to encounter mismatches between the measured spectra and the theoretical predictions. In those cases, it is common practice to rescale the calculated spectrum by a constant scaling factor for better agreement with the experiment [28]. For example, in Ref. [26] harmonic frequencies of $\mathrm{Au}_{n} \mathrm{Kr}(n=7,19,20)$ clusters are scaled by a constant factor of 1.15. In Ref. [21], no scaling factor was used for $\mathrm{Au}_{n} \mathrm{Ar}_{m}^{+}(n \leq 5 ; m \leq 5)$, whereas a factor of 1.04 was applied for $n>6$. While applying a certain theory level in combination with a scaling factor can provide a good experiment-theory agreement for infrared spectra, such empiric correction does not guarantee a proper description of the interaction between the metal ion and the noble gas atoms.

Despite the efforts, the interaction between metal atoms and small clusters with noble gases is still the subject of intensive research, in particular from the theoretical side [29, 30, 31, 32]. While theoretical studies require a proper benchmark analysis to select the level of theory, there is a major lack of experimental reference data. To the best of our knowledge, experimentally determined values are only available for the binding energies of $\mathrm{AgAr}^{+}$(lower limit; < $0.31 \mathrm{eV}$ ) [33] and $\mathrm{Au}_{2} \mathrm{Ar}^{+}$(rough estimation of $0.1 \mathrm{eV}$ ) [34]. Therefore, most theoretical studies rely solely on comparisons with high-level computational simulation methods, such as coupled-cluster calculations.

In this work, we have measured infrared spectra of a variety of small $\mathrm{MAr}_{n}{ }^{+}(n=3-5 ; M=\mathrm{Au}, \mathrm{Ag}, \mathrm{Pd})$ complexes by infrared multiple photon dissociation (IRMPD) spectroscopy. These spectra are used as benchmark for computations with different generations of density functional theory (DFT). The purpose of this work is to illustrate the method selection for calculations on $\mathrm{MAr}_{n}{ }^{+}$ complexes and to give advice about how to select a computational level in future studies. Although the bonding mechanism determines the infrared spectra of the $\mathrm{MAr}_{n}{ }^{+}$ complexes, a thorough analysis of the chemical interactions goes beyond the scope of this article.

\section{Methods}

\subsection{Experiment}

$\operatorname{MAr}_{n}{ }^{+}(M=\mathrm{Au}, \mathrm{Ag}, \mathrm{Pd})$ complexes are produced in a laser ablation source that has been developed for the production of metal clusters and is described in detail elsewhere [35]. Briefly, metal targets are ablated by a focused pulsed laser beam (Nd:YAG laser, $10 \mathrm{~Hz}$ ). The ablated plasma is cooled by a pulse of helium gas (backing pressure of 8 bar) and expands supersonically into vacuum through a conical nozzle. The source is kept at $200 \mathrm{~K}$ by a continuous flow of liquid $\mathrm{N}_{2}$. The Ar complexes are formed by mixing $2 \% \mathrm{Ar}$ in the $\mathrm{He}$ carrier gas. After complex formation, the molecular beam is collimated before entering a reflectron time-of-flight mass spectrometer. The vibrational modes of the complexes are characterized by far-infrared multiphoton dissociation (IRMPD) experiments [36], performed by overlapping the collimated molecular beam with the light of the free electron laser FELICE, adapted for intracavity experiments [37]. FELICE was operated in the $90-240 \mathrm{~cm}^{-1}$ spectral range in $1 \mathrm{~cm}^{-1}$ steps and at a repetition rate of $5 \mathrm{~Hz}$. Infrared spectra are recorded by comparing metal-Ar complex intensities in mass spectra with $(I)$ and without $\left(I_{0}\right)$ laser interaction. The IRMPD signal is defined as $Y_{\mathrm{IR}}=-$ $\log \left(I_{0}\right) / F$, with $F$ the fluence of FELICE. In order to increase the signal-to-noise ratio of the measured spectra, FELICE was slightly defocused to optimize the overlap of the laser light with the molecular beam.

Under the applied experimental conditions, complexes with several Ar atoms are formed. We concentrate on complexes for which infrared spectra with a high signal-tonoise ratio can be measured, i.e. complexes with a high intensity in mass spectra and for which the IRMPD signal is not contaminated by the fragmentation of complexes with more attached Ar atoms [21]. In addition, the dissociation energy of the complex must be low enough that the laser excitation induces Ar loss within the time scale of the experiment.

\subsection{Calculations}

Density functional theory calculations are performed with the NWChem 6.8 [38] software package. Different exchange-correlation functionals were employed: (1) GGA PBE, (2) meta-GGA TPSS, (3) hybrid B3LYP, (4) long range-corrected LC-wPBEh and (5) double hybrid B2PLYP. The functionals in this selection are fundamentally different in nature and represent different levels of complexity within Perdew's Jacob's Ladder [39]. PBE is a GGA functional, depending on the electron density and its gradient [40]. TPSS is a meta-GGA functional, which also includes the second derivative of the electron density [41]. B3LYP is probably the most common hybrid 
functional, it adds a fraction of exact exchange from Hartree-Fock to the energy [42]. The LC-wPBEh functional belongs to the range-separated hybrid functionals, which aim to correct the wrong asymptotic behavior of the effective potential in DFT [43]. Finally, the B2PLYP functional belongs to the double-hybrid category, which, in addition to a fraction of exact exchange from Hartree-Fock, includes a portion of exact correlation by performing a MP2 correction [44]. Due to the required MP2 step, doublehybrid functionals computationally are more demanding than functionals lower on Perdew's Jacob's Ladder. Nevertheless, double hybrids are thought to provide the most accurate energy calculations within the DFT formalism. Despite the considerable differences in how these functionals are constructed, almost all of them have recently been used in $\mathrm{MAr}_{n}{ }^{+}$calculations: $\mathrm{PBE}$ was used to determine the structures of $\mathrm{Au}_{n}{ }^{+}(n=3-9)$ with $\mathrm{Ar}$ as tagging atoms [21]. TPSS was applied in an IRMPD study determining the structures of $\mathrm{Ag}_{n}{ }^{+}$clusters, also using $\mathrm{Ar}$ as tagging atom [22]. B3LYP was used in a theoretical study of $\mathrm{AuAr}_{n}{ }^{+}$complexes [12]. Finally, LC-wPBEh was selected in studies addressing the interaction of small bimetallic silver-gold clusters with Ar [19, 20].

For the calculations using these different functionals, the Def2-TZVPP basis set was employed together with the Stuttgart Def2-ECP pseudopotentials for $\mathrm{Au}, \mathrm{Pd}$ and $\mathrm{Ag}$ [45] which treat explicitly 19, 18 and 19 valence electrons. Scalar relativistic effects are included in these ECPs. The effect of basis set length was investigated by comparing the Def2-SVP, Def2-TZVPP and Def2-QZVPP basis sets [45]. The effect of including dispersion forces was studied by performing calculations using the D3BJ dispersioncorrection [46]. Calculated infrared spectra were constructed without applying a scaling factor and assuming a Gaussian profile for each vibrational mode with a width of $5 \mathrm{~cm}^{-1}$. An isomer search is performed by randomly positioning the Ar atoms around the metal ion. Bader partial charges and Wiberg bond indexes have been calculated using the Multiwfn software package [47]. Wiberg bond indexes correspond to the average number of electron pairs shared by two atoms, it ranges from 0 to 1 , with a large value indicating covalent interaction.

\section{Results and discussion}

At first, a search for low-lying isomers was performed for the investigated complexes, by randomly positioning the Ar atoms around the metal ion. Next, each complex structure was locally reoptimized at the different levels of theory and their vibrational frequencies calculated; thereafter, the computed spectra were compared with the measured IRMPD spectra. The located putative lowest-energy structures are presented in Fig. 1, using TPSS for $\mathrm{AuAr}_{3}{ }^{+}$ and $\mathrm{AuAr}_{4}{ }^{+}, \mathrm{PBE}$ for $\mathrm{AgAr}_{4}{ }^{+}$and LC-wPBEh for $\mathrm{PdAr}_{5}{ }^{+}$; an explanation for this selection is given in section 3.2. In Figure 1, bond lengths and Bader partial charges are given.
While the $\mathrm{AgAr}_{4}{ }^{+}$and $\mathrm{PdAr}_{5}{ }^{+}$complexes adopt high symmetry geometries $\left(\mathrm{T}_{\mathrm{d}}\right.$ and $\left.\mathrm{C}_{4 \mathrm{v}}\right), \mathrm{AuAr}_{3}{ }^{+}$and $\mathrm{AuAr}_{4}{ }^{+}$have lower symmetry $\left(\mathrm{C}_{2 \mathrm{v}}\right)$. $\mathrm{AuAr}_{3}{ }^{+}$is planar but the $\mathrm{Ar}-\mathrm{Au}-\mathrm{Ar}$ angles differ from $120^{\circ}$. Similar calculations were performed for $\mathrm{AuAr}_{n}{ }^{+}$clusters, using the B3LYP functional [12]. Interestingly, when applying different functionals for the calculations, bond lengths and bond angles vary, in some cases to a large extent (see section 3.2), consequently modifying the infrared spectra, as detailed in the following.

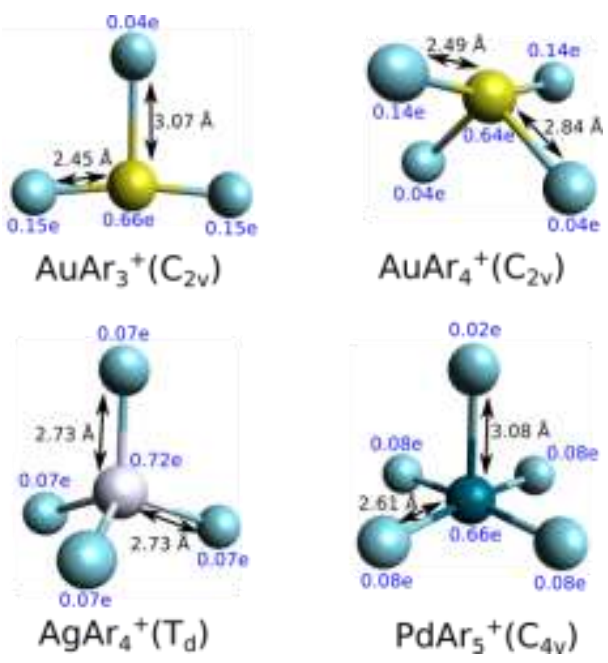

Figure 1. Optimized structures of $\mathrm{AuAr}_{3}{ }^{+}$(TPSS), $\mathrm{AuAr}_{4}{ }^{+}$(TPSS), $\mathrm{AgAr}_{4}{ }^{+}$(PBE) and $\mathrm{PdAr}_{5}{ }^{+}$(LC-wPBEh). Gold atoms are shown in yellow, silver in light-grey, palladium in blue, and argon in cyan. Bond lengths (in $\AA$ ) and Bader partial (in e) are given. The point group is listed between parentheses.

The experimental IR spectra of $\mathrm{AuAr}_{3}{ }^{+}, \mathrm{AuAr}_{4}{ }^{+}$, $\mathrm{AgAr}_{4}{ }^{+}$, and $\mathrm{PdAr}_{5}{ }^{+}$are presented in Fig. 2. Within the recorded wavenumber range $\left(90-240 \mathrm{~cm}^{-1}\right)$ all the complexes show a single intense infrared absorption band, whose center is determined by a Gaussian fit and listed in Fig. 2.

\subsection{Varying the basis set size}

The role of the basis set size was investigated for a fixed functional, using as reference the measured infrared spectra. Three basis sets from the Ahlrichs Def2 family were employed for this purpose: split valence Def2-SVP, triplezeta valence Def2-TZVPP and quadruple-zeta valence Def2-QZVPP [45]. The analysis for $\mathrm{AuAr}_{3}{ }^{+}$and $\mathrm{AuAr}_{4}{ }^{+}$is presented in Figs. 2(a) and 2(b), where the TPSS functional was employed (this choice is discussed in section 3.2). The small Def2-SVP basis set gives poor results. For $\mathrm{AuAr}_{3}{ }^{+}$the mode observed at $216 \mathrm{~cm}^{-1}$ is predicted at $180 \mathrm{~cm}^{-1}$, and a second calculated mode close to $100 \mathrm{~cm}^{-1}$ is not observed in the experimentally probed range. Instead, the much larger Def2-QZVPP gives a single mode within the measured wavenumber range, which is located at $212 \mathrm{~cm}^{-1}$, thus off by only $4 \mathrm{~cm}^{-1}$. The second mode is calculated at $63 \mathrm{~cm}^{-1}$ and consequently not observed in the measured frequency range. The differences between Def2-TZVPP and Def2-QZVPP 
are minor, although the latter gives a slightly better agreement. As expected, the computation time increases with the basis set size; Def2-SVP calculations are roughly twice faster than those with Def2-TZVPP, which in turn are twice as fast as the Def2-QZVPP calculations. Similar results are obtained for $\mathrm{AuAr}_{4}{ }^{+}$, with a large predicted mismatch of $63 \mathrm{~cm}^{-1}$ using the Def2-SVP basis set, and the appearance of two extra modes that are not found in the experiment. The mismatch of the main mode reduces to 4 $\mathrm{cm}^{-1}$ with Def2-TZVPP and to $1 \mathrm{~cm}^{-1}$ for Def2-QZVPP, while other modes have a much lower wavenumber.

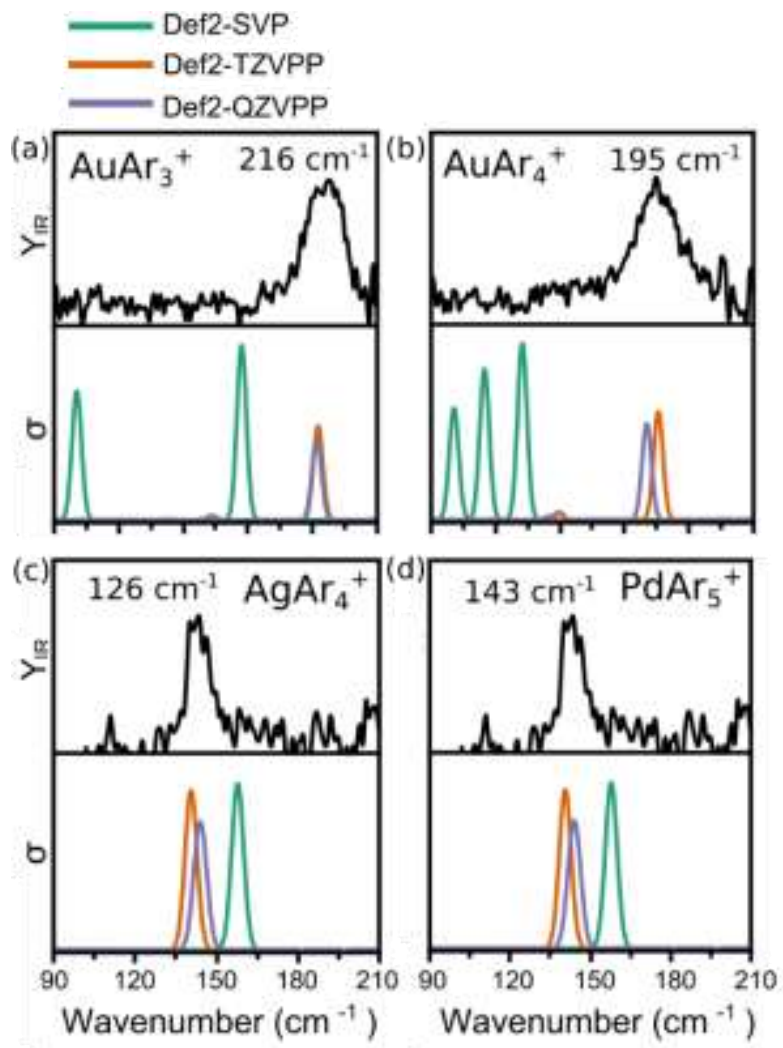

Figure 2. Experimental and calculated IR spectra of a) $\mathrm{AuAr}_{3}{ }^{+}$ (TPSS), b) $\mathrm{AuAr}_{4}^{+}$(TPSS), c) $\mathrm{AgAr}_{4}{ }^{+}$(PBE) and d) $\mathrm{PdAr}_{5}{ }^{+}$(LCwPBEh). The experimentally determined centers of the vibrational modes are given above the measured IRMPD spectra. Three basis sets are employed for the calculations, Def2-SVP (green), Def2TZVPP (orange) and Def2-QZVPP (purple).

These observations are independent of the selected functional. All functionals perform significantly worse with the very small Def2-SVP basis set than with Def2-TZVPP. The performance can be quantified by calculating a functional-averaged deviation coefficient $\left(\chi_{\text {basis }}\right)$ for each basis set, which accounts for the frequency deviation of the mode at the highest frequency for all the five tested functionals for a specific cluster. It is defined as

$$
\chi_{\text {basis }}=\sqrt{\sum_{i}\left(v_{D F T, i}-v_{\text {exp }}\right)^{2}} / n_{f u n c}
$$

where $v_{D F T, i}$ represents the calculated frequency obtained using functional $i, v_{\text {exp }}$ is the measured frequency and $n_{\text {func }}$ the number of functionals evaluated, here five. Thus, a smaller $\chi_{\text {basis }}$ represents a functional-averaged better agreement between experiment and theory with a specific basis set. Using the Def2-SVP basis set, $\chi_{\text {svp }}$ is $20 \mathrm{~cm}^{-1}$ for $\mathrm{AuAr}_{3}{ }^{+}$and $33 \mathrm{~cm}^{-1}$ for $\mathrm{AuAr}_{4}{ }^{+}$, whereas with Def2-TZVPP the values are 8 and $21 \mathrm{~cm}^{-1}$. Further improvement is found with Def2-QZVPP, giving values of 3 and $7 \mathrm{~cm}^{-1}$. The coefficient becomes smaller (and thus better) when increasing the basis set size.

A similar analysis was performed for the $\mathrm{AgAr}_{4}{ }^{+}$and $\mathrm{PdAr}_{5}{ }^{+}$complexes, using the PBE and LC-wPBEh functionals, respectively (see section 3.2 for motivation of this choice). The analysis is presented in the bottom panels of Fig. 2. Although in these cases the mismatches with the Def2-SVP basis set are less marked, again the very small basis set is insufficient, considering the large $\chi_{S V P}$ values of $26 \mathrm{~cm}^{-1}$ for $\mathrm{AgAr}_{4}{ }^{+}$and $33 \mathrm{~cm}^{-1}$ for $\mathrm{PdAr}_{5}{ }^{+}$. Instead, using Def2-TZVPP gives smaller values of 10 and $4 \mathrm{~cm}^{-1}$. With Def2-QZVPP $\chi_{\text {basis }}$ decreases to $8 \mathrm{~cm}^{-1}$ for $\mathrm{AgAr}_{4}{ }^{+}$and 4 $\mathrm{cm}^{-1}$ for $\mathrm{PdAr}_{5}{ }^{+}$. Similar results are obtained in these cases by employing the Def2-TZVPP and Def2-QZVPP basis sets, while the latter requires significantly longer computational times (e.g. four times longer for $\mathrm{PdAr}_{5}{ }^{+}$).

Therefore, we conclude that independently on the metal ion, the small Def2-SVP basis, irrespective of the selected functional, poorly predicts the vibrational spectra of the Ar complexes. A triple zeta basis set is at least required. Considering the computer costs of larger basis sets, Def2TZVPP or equivalent basis sets could be a good compromise.

\subsection{Varying the exchange-correlation functional}

In view of the satisfactory performance of Def2-TZVPP, this basis set was selected for evaluating different exchangecorrelation functionals. Among the many functionals that are nowadays available in DFT software packages [48], we selected five popular ones: PBE, TPSS, B3LYP, LC-wPBEh and B2PLYP.

The IR spectra of the $\mathrm{AuAr}_{3}{ }^{+}$(left) and $\mathrm{AuAr}_{4}{ }^{+}$(right) complexes calculated with the different functionals are compared with the IRMPD spectra in Fig. 3. For $\mathrm{AuAr}_{3}{ }^{+}$, two bands are predicted within the measured spectral range, although one of these has a low intensity. These correspond to the symmetric and anti-symmetric stretching of the nearly collinear Ar-Au-Ar atoms. All functionals perform relatively well, although the band calculated with B3LYP is clearly red-shifted. The differences between the other four functionals are small, with TPSS $\left(213 \mathrm{~cm}^{-1}\right)$ being the closest to the experimental value. The agreement is more functional-dependent for $\mathrm{AuAr}_{4}{ }^{+}$, where the main band corresponds to the anti-symmetric Ar-Au-Ar stretching represented by red arrows in Fig. 4. In particular, the B3LYP calculated frequency is strongly red-shifted and there is a 
second intense mode close to $90 \mathrm{~cm}^{-1}$. The PBE-calculated band is also red-shifted, although not as dramatic as for B3LYP. The TPSS prediction $\left(195 \mathrm{~cm}^{-1}\right)$ is again the best, followed by B2PLYP $\left(188 \mathrm{~cm}^{-1}\right)$.

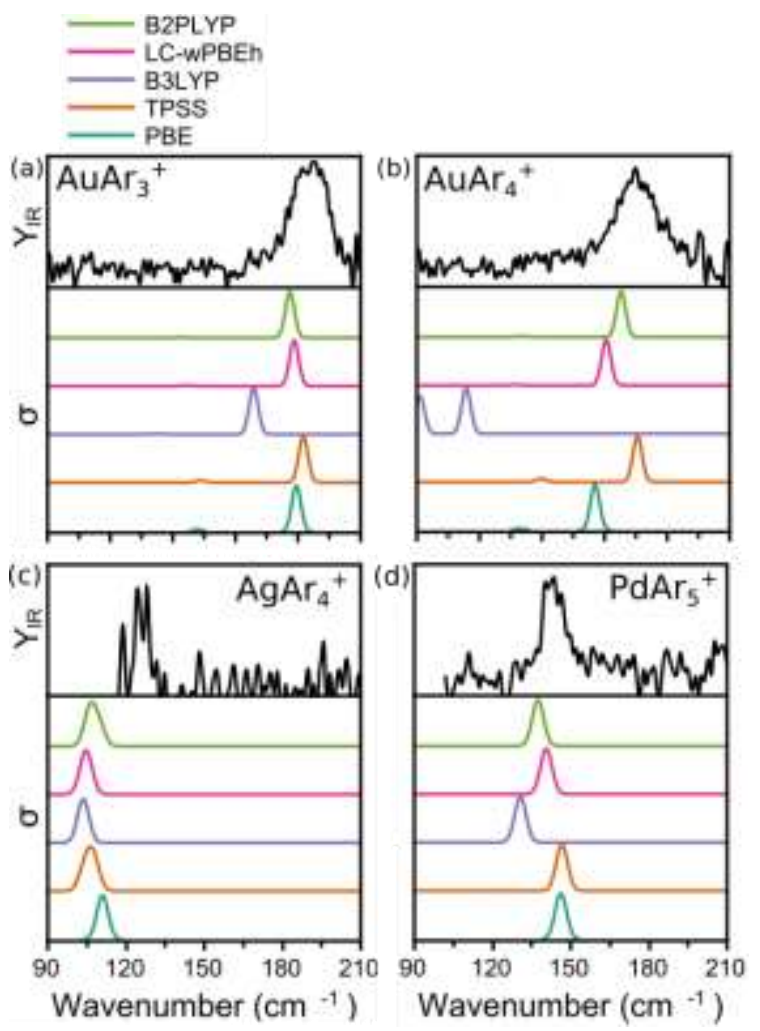

Figure 3. Experimental and calculated IR spectra of a) $\mathrm{AuAr}_{3}{ }^{+}, \mathrm{b}$ ) $\mathrm{AuAr}_{4}{ }^{+}$, c) $\mathrm{AgAr}_{4}{ }^{+}$and d) $\mathrm{PdAr}_{5}{ }^{+}$. Calculations are performed with the Def2-TZVPP basis set and the B2PLYP (light green), LCwPBEh (magenta), B3LYP (purple), TPSS (orange), and PBE (green) functionals.

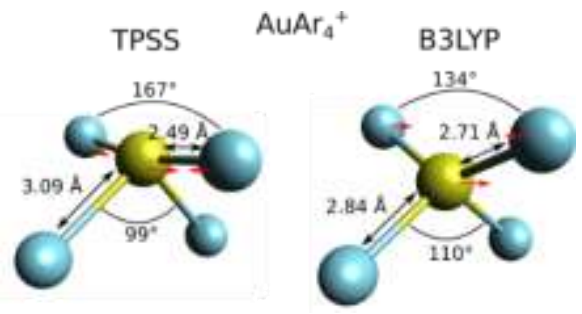

Figure 4. Comparison of the structures of $\mathrm{AuAr}_{4}{ }^{+}$computed with the TPSS and B3LYP functionals. Bond lengths and bond angles are presented for both cases. The displacement of the atoms for the main vibrational mode are represented by red arrows.

The large disagreement between theory and experiment that is obtained by using B3LYP can be explained by both a wrong geometry prediction and a bad description of the potential energy surface. In Fig. 4 we observe how the geometry of $\mathrm{AuAr}_{4}{ }^{+}$optimized with the B3LYP functional differs from the one with TPSS. Their bond lengths and bond angles are clearly different between both methods, in line with the considerably different infrared spectra. Repeated calculations using the ORCA 4.2 software package [49] yielded the same results. Additional experimental information, such as bond lengths or $\mathrm{Ar}$ binding energies, would be helpful to enhance understanding, but these data are currently unavailable. The differences in the calculated geometries are less severe for $\mathrm{AuAr}_{3}{ }^{+}$, and even less for $\mathrm{AgAr}_{4}{ }^{+}$and $\mathrm{PdAr}_{5}{ }^{+}$.

In the bottom panels of Fig. 3, a similar analysis is presented for $\mathrm{AgAr}_{4}{ }^{+}$(left panel) and $\mathrm{PdAr}_{5}{ }^{+}$(right) panel. For $\mathrm{AgAr}_{4}{ }^{+}$, the variation between the tested functionals is relatively small; all functionals predict a vibrational mode red-shifted with respected to the experimental value. The best agreement is found for PBE, although differences with other functionals are marginal. For $\mathrm{PdAr}_{5}{ }^{+}$, both $\mathrm{PBE}$ and TPSS predict a slightly blue-shifted band with respect to the experiment, in contrast to the slight red shifts for B3LYP, LC-wPBEh and B2PLYP. The best prediction now comes from LC-wPBEh $\left(141 \mathrm{~cm}^{-1}\right)$, with other functionals yielding somewhat larger deviations. Interestingly, the pronounced mismatch of the B3LYP predicted frequencies observed for $\mathrm{AuAr}_{3}{ }^{+}$and especially for $\mathrm{AuAr}_{4}{ }^{+}$is not observed for $\mathrm{AgAr}_{4}{ }^{+}$, although it provides the least accurate prediction.

To quantify the agreement for the four investigated systems, we define a deviation coefficient for each functional $\left(\chi_{\text {func }}\right)$, which accounts for the frequency deviation of the calculated spectra for a specific functional:

$$
\chi_{f u n c}=\sqrt{\sum_{j}\left(v_{D F T, j}-v_{\text {exp }}\right)^{2}} / n_{\text {complex }} .
$$

Here $v_{D F T, j}$ is the calculated frequency of complex $j$ and $n_{\text {complex }}$ is the number of investigated complexes (i.e. four). Using expression (2), the $\chi_{\text {func }}$ values are: $7 \mathrm{~cm}^{-1}$ (PBE), 5 $\mathrm{cm}^{-1}$ (TPSS), $23 \mathrm{~cm}^{-1}$ (B3LYP), $7 \mathrm{~cm}^{-1}$ (LC-wPBEh) and 7 $\mathrm{cm}^{-1}$ (B2PLYP). Therefore, accounting for all the investigated systems, TPSS agrees better, although the simpler and less computer demanding PBE gives a similar accord. The popular B3LYP functional deviates strongly from the experiment.

\subsection{Inclusion of dispersion correction}

Including dispersion forces in the calculations can have a remarkable effect when computing the infrared spectra of the complexes. For example, as shown in the previous analysis, none of the considered functionals yields a satisfactory agreement between the calculations and the experiment for $\mathrm{AgAr}_{4}{ }^{+}$, even when using the larger, and more expensive, Def2-QZVPP basis set (see Fig. 2). Nonetheless, including the D3BJ correction to the PBE functional in conjunction with the Def2-QZVPP basis set, reduces the redshift from 12 to $9 \mathrm{~cm}^{-1}$. This is also the case for the Au complexes. Taking the PBE functional as example, the predicted vibrational modes were off by 7 for $\mathrm{AuAr}_{3}{ }^{+}$and by $20 \mathrm{~cm}^{-1}$ for $\mathrm{AuAr}_{4}{ }^{+}$. Including the D3BJ correction the deviations strongly reduce to 2 and $1 \mathrm{~cm}^{-1}$, respectively (see Fig. 5). 


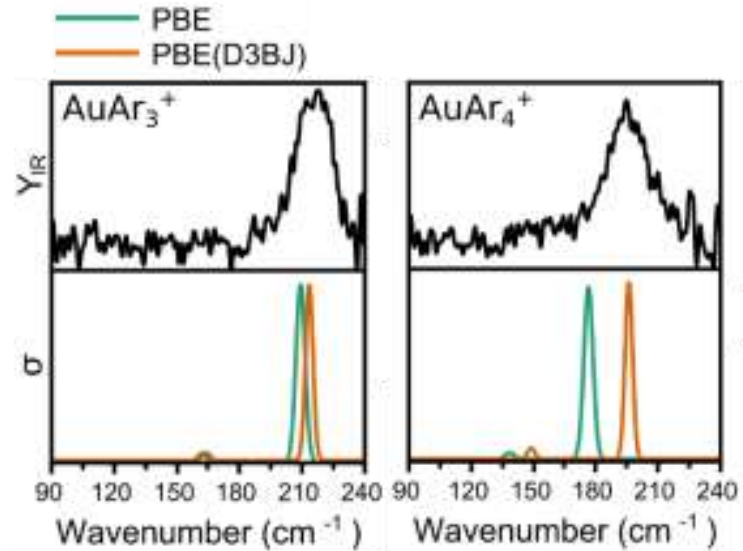

Figure 5. Top: IR spectrum measured for the $\mathrm{AuAr}_{3}{ }^{+}$(left) and $\mathrm{AuAr}_{4}{ }^{+}$(right) complexes. Bottom: Vibrational spectra of the complexes calculated with the Def2-TZVPP basis set and the PBE functional with (orange) and without (green) dispersion corrections.

The interaction between one gold atom and one argon atom in $\mathrm{AuAr}^{+}$is of substantial covalent character. The complexity of the interaction increases when adding $\mathrm{Ar}$ atoms. The additional $\mathrm{Ar}$ atoms interact weaklier with $\mathrm{Au}^{+}$. In addition, the Ar-Ar interactions become relevant. The electron densities of the $\mathrm{AuAr}_{3}{ }^{+}$and $\mathrm{AuAr}_{4}{ }^{+}$complexes, shown in Fig. 6, represent the interactions of each Ar atom with the metal. Both $\mathrm{AuAr}_{3}{ }^{+}$and $\mathrm{AuAr}_{4}{ }^{+}$have two Ar atoms with a substantial electron density in between $\mathrm{Ar}$ and $\mathrm{Au}$, reflecting a substantial covalent character of their interactions. This result agrees with the high $\mathrm{AuAr}_{2}{ }^{+}$ intensity in mass spectra [16]. For the additional Ar atoms there is no $\mathrm{Ar}-\mathrm{Au}$ electron density overlap.

This is reflected in the adsorption energies of the $n^{\text {th }}$ attached $\mathrm{Ar}$ atom in $\mathrm{AuAr}_{n}{ }^{+}$, calculated as:

$$
E_{a d s}=E\left(\operatorname{MAr}_{n}^{+}\right)-E\left(\operatorname{MAr}_{n-1}{ }^{+}\right)-E(\mathrm{Ar}),
$$

where $E$ is the zero-point corrected total energy of the species in parenthesis $(M=\mathrm{Au}, \mathrm{Ag}$ or $\mathrm{Pd}) . \mathrm{For}_{\mathrm{AuAr}}{ }^{+}$with $n$ from 1 to 4 , the $E_{\text {ads }}$ values are $-0.53,-0.68,-0.05$ and $0.06 \mathrm{eV}$ (at TPSS level). We note that the adsorption energy of the second adsorbed Ar atom is higher than that of the first; such effect has been seen before for $\mathrm{AuAr}_{n}{ }^{+}$and $\mathrm{CuAr}_{n}^{+}$complexes [16, 50]. A much lower adsorption energy is observed from the third $\mathrm{Ar}$ atom onwards, corresponding to weaker bound $\mathrm{Ar}$ atoms, with the interaction dominated by dispersion forces instead of a covalent-type of bond. To support this conclusion, Wiberg bond indexes were calculated for $\mathrm{AuAr}_{3}{ }^{+}$and $\mathrm{AuAr}_{4}{ }^{+}$. For $\mathrm{AuAr}_{3}{ }^{+}$, the $\mathrm{Ar}-\mathrm{Au}$ bonds with the two symmetric Ar atoms has a value of 0.8 , reflecting an interaction of substantial covalent nature. The index for the third $\mathrm{Ar}$ atom and $\mathrm{Au}$ is only 0.3 . For $\mathrm{AuAr}_{4}{ }^{+}$there are two types of bonds, with 0.74 and 0.34 indexes, also in agreement with the electron density results. The presence of these weakly attached Ar atoms is likely the cause of inaccurate predictions of bond lengths and bond angles (and consequently of the vibrational spectrum) by some DFT functionals that incorrectly model dispersion. Although adsorption energies for $\mathrm{AuAr}_{n}{ }^{+}$ complexes have not been so far determined experimentally, we can compare our calculations with values computed using wave function based post-Hartree-Fock methods, such as MP2 and $\operatorname{CCSD(T).~In~Ref.~[7],~} E_{a d s}$ was calculated for $\mathrm{AuAr}^{+}$, obtaining -0.54 and $-0.48 \mathrm{eV}$ at the MP2 and $\operatorname{CCSD}(\mathrm{T})$ levels, respectively. We obtain values of -0.62 , $0.53,-0.43$ and $-0.41 \mathrm{eV}$ for PBE, TPSS, B3LYP and B2PLYP (using the Def2-TZVPP basis set and dispersion correction). Therefore, TPSS seems to get closer to the postHartree-Fock calculations.

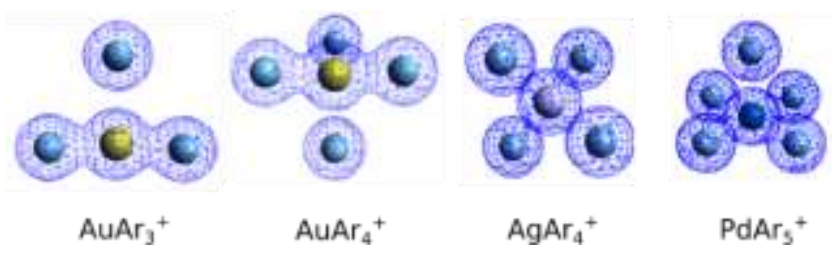

Figure 6. Electron density plots of the $\mathrm{AuAr}_{3}{ }^{+}, \mathrm{AuAr}_{4}{ }^{+}, \mathrm{AgAr}_{4}{ }^{+}$ and $\mathrm{PdAr}_{5}{ }^{+}$complexes. The density is plotted at $0.02 \mathrm{e}^{-3}$.

In previous studies it was shown that most exchangecorrelation functionals fail to describe correctly the dispersion interaction between closed-shells atoms and molecules, such as in the $\mathrm{Kr}-\mathrm{Kr}$ dimer [51]. While a reference $\operatorname{CCSD}(\mathrm{T})$ calculation predicts a bound state between both atoms, B3LYP yields a purely repulsive interaction, thus drastically failing in describing the dispersion interaction. PBE instead, computes a bound state, although of lower binding energy than the reference calculation. The three semi-empirical coefficients in the B3LYP hybrid functional have been determined on the basis of a data set composed mainly of organic species [52], what could explain why it is less suited for transition metal ion noble gas complexes.

The electron density in $\mathrm{AgAr}_{4}{ }^{+}$, in contrast to $\mathrm{AuAr}_{4}{ }^{+}$, indicates a similar interaction of all the $\mathrm{Ar}$ atoms with $\mathrm{Ag}^{+}$, with non-zero electron density in the region between Ar and $\mathrm{Ag}$, thus revealing a much simpler interaction. All four bonds have Wiberg indexes of 0.51 , which is smaller than for the first two $\mathrm{Ar}$ atoms in $\mathrm{AuAr}_{3}{ }^{+}$and $\mathrm{AuAr}_{4}{ }^{+}$. The adsorption energies of subsequent $\mathrm{Ar}$ atoms in $\mathrm{Ag}^{+}$vary more gradually than for gold, being $-0.43,-0.43,-0.24$ and $0.21 \mathrm{eV}$ for $n$ from 1 to 4 (PBE/Def2-QZVPP/D3 level). Therefore, the first two Ar atoms are weaklier bound to $\mathrm{Ag}^{+}$ than to $\mathrm{Au}^{+}$, but in general the interaction of all Ar atoms with $\mathrm{Ag}^{+}$is similar. Also, in the $\mathrm{Ag}^{+}$case the $E_{\text {ads }}$ decreases with every additional Ar atoms, since less electron density can be transferred from $\mathrm{Ar}$ to $\mathrm{Ag}^{+}$, but this decrease is less pronounced than for the $\mathrm{Au}^{+}$complexes. Previous CCSD(T) calculations of the $\mathrm{AgAr}^{+}$dimer have found a dissociation energy of $0.44 \mathrm{eV}$ [53], in perfect agreement with our calculation. For $\mathrm{PdAr}_{n}^{+}$, the first four attached Ar atoms 
interact more strongly; the adsorption energies for $n$ from 1 to 5 are $-0.60,-0.54,-0.23,-0.28$ and $-0.07 \mathrm{eV}$ (PBE/Def2TZVPP/D3). Thus, the bonding mechanisms in these complexes is more evolved than in the Ag case, although still simpler than for the $\mathrm{Au}$ species. $\mathrm{PdAr}_{5}{ }^{+}$has two different types of bonds with Wiberg indexes of 0.62 (for the bond of the four Ar atoms in the same plane as the Pd atom) and 0.31 (for the bond of the Ar atom outside this plane). These indexes are consistent with the conclusions from the electron density plot.

Table 1. Deviation coefficient $\chi_{\text {func }}$ calculated for each functional and the Def2-SVP and Def2-TZVPP basis sets, the later with and without the inclusion of dispersion corrections. $\chi_{\text {comp }}$ is presented in $\mathrm{cm}^{-1}$.

\begin{tabular}{lccc}
\hline \hline & \multicolumn{3}{c}{$\boldsymbol{\chi}_{\text {func }}$} \\
\hline \hline PBE & Def2-SVP & Def2-TZVPP & Def2-TZVPP/D3 \\
\hline TPSS & 18 & 7 & 3 \\
\hline B3LYP & 18 & 5 & 4 \\
\hline LC-wPBEh & 24 & 23 & 14 \\
\hline B2PLYP & 15 & 7 & - \\
\hline \hline
\end{tabular}

We finally analyze the performance of the different functionals throughout the investigated complexes, by presenting a comparison of the $\chi_{\text {func }}$ parameters calculated with the Def2-SVP and Def2-TZVPP basis sets. For the second basis set, values are also presented by including dispersion corrections. As discussed above, the small Def2SVP basis set yields poor agreement with the experiments, while Def2-TZVPP performs much better. By including dispersion corrections an even better agreement is obtained for all functionals. In NWChem, the software employed in this study, D3BJ corrections are not implemented in conjunction with the LC-wPBEh functional. The functional (and software) has also been used in previous investigations of $\mathrm{Au}_{n} \mathrm{Ar}^{+}(n=3-5)$ clusters, without employing D3BJ corrections $[19,20]$. Our analysis shows that the best agreement with the spectroscopy experiment is found when such corrections are applied. Based on the data in Table 1, the PBE/Def2-TZVPP/D3 theory level seems appropriate for calculating properties of small $M^{+}-\mathrm{Ar}_{\mathrm{n}}$ complexes. Considering the speed of the calculations employing PBE, and the accuracy achieved when both Def2-TZVPP and D3BJ are used. We remark, however, that the use of scaling factors can correct for small mismatches in the prediction of IR spectra. Nevertheless, based on our calculated adsorption energies and the existing $\operatorname{CCSD}(\mathrm{T})$ values of selected complexes, such proposed theory level seems adequate to describe $M^{+}-\mathrm{Ar}_{n}$ complexes.

\section{Conclusions}

We reported infrared spectra of $\operatorname{MAr}_{n}{ }^{+}(n=3-5 ; M=\mathrm{Au}$, $\mathrm{Ag}, \mathrm{Pd})$ and used them to perform a benchmark analysis of different theory levels within the density functional theory formalism. The size of the basis set, different exchange- correlation functionals and the inclusion of dispersion correction were explored. Our results show a strong dependence of the obtained agreement between theory and experiment on the employed level of theory. Independently of the employed functional, the smallest basis set yields large mismatches between theory and experiment. In addition, a proper selection of the used functional is important for the calculations, with more complex (and computationally more demanding) functionals not necessarily giving better results. In particular, the B3LYP functional, widely used for systems containing lighter elements, performs poor in comparison with the simpler (and faster) PBE functional. Including dispersion corrections improves the theory-experiment agreement, independently of the employed functional. Our analysis suggests that the PBE/Def2-TZVPP/D3 theory level is appropriate for calculations of $M^{+}-\mathrm{Ar}_{\mathrm{n}}$ complexes, requiring none or only small scaling factors when computing IR spectra. This level yields adsorption energies close to the values obtained by the expensive $\operatorname{CCSD}(\mathrm{T})$ theory.

\section{Acknowledgements}

This work has been supported by the KU Leuven Research Council (project C14/18/073) and by the CALIPSOplus project, under the Grant Agreement 730872 from the EU Framework Programme for Research and Innovation HORIZON 2020. LDC is supported by King's College London through the NMS Faculty Studentship Scheme. PF acknowledges the Research Foundation - Flanders (FWO) for a postdoctoral grant. The work has been performed under the Project HPC-EUROPA3 (INFRAIA-2016-1-730897), with the support of the EC Research Innovation Action under the H2020 Programme; in particular, the author gratefully acknowledges the support of the Physics Department at King's College London and the computer resources and technical support provided by EPCC at The University of Edinburgh. We thank the financial support offered by the Royal Society (No. RG 120207) via the membership of the UK's HEC Materials Chemistry Consortium, which is funded by the EPSRC (EP/L000202). We are grateful to the UK Materials and Molecular Modelling Hub for computational resources, which is partially funded by EPSRC (EP/P020194/1). This work used the ARCHER UK National Supercomputing Service. We gratefully acknowledge the Nederlandse Organisatie voor Wetenschappelijk Onderzoek (NWO) for the support of the FELIX Laboratory and thank the FELIX staff.

\section{References}

[1] S. Pan, G. Jana, B. Merino and P. K. Chattaraj, ChemistryOpen, 8, 173-187, 2019.

[2] W. Grochala, Soc. Rev., 36, 1632-1655, 2007.

[3] S. Kapur and E. W. Müller, Surf. Sci., 62, 610-620, 1977.

[4] P. Pyykkö, J. Am. Chem. Soc., 117, 2067-2070, 1995.

[5] J. P. Read and A. D. Buckingham, J. Am. Chem. Soc., 119, 9010-9013, 1997. 
[6] D. Bellert and W. H. Breckenridge, Chem. Rev., 102, 15951622, 2002.

[7] L. Belpassi, I. Infante, F. Tarantelli and L. Visscher, J. Am. Chem. Soc., 1303, 1048-1060, 2008.

[8] C. W. Bauschlicher Jr., H. Partridge and S. R. Langhoff, Chem. Phys. Lett., 165, 272-276, 1990.

[9] J. V. Burda, N. Runeberg and P. Pyykkö, Chem. Phys. Lett., 288, 635-641, 1998.

[10] Y. Taketsugu, T. Taketsugu and T. Noro, J. Chem. Phys., 125, 154308, 2006.

[11] N. R. Walker, L. M. Reynard and M. C. L. Gerry, J. Mol. Struct., 612, 109-116, 2002.

[12] P. Zhang, Y. Zhao, F. Hao, X. Song, G. Zhang and Y. Wang, J. Mol. Struc-Theochem., 899, 111-116, 2009.

[13] E. Eliav, U. Kaldor and Y. Ishikawa, Phys. Rev. A, 49, 1724, 1994.

[14] P. Pyykkö, Chem. Rev., 88, 563-594, 1988.

[15] P. Pyykkö, Angew. Chem. Int. Ed., 43, 4412-4456, 2004

[16] P. Martini, L. Kranabetter, M. Goulart, B. Rasul, M. Gatchell, P. Scheier and O. Echt, Phys. Chem. A, 123, 9505-9513, 2019.

[17] M. Mahmoodi-Darian, P. Martini, L. Tiefenthaler, J. Kočišek, P. Scheier and O. Echt, J. Phys. Chem. A, 123, 10426-10436, 2019.

[18] V. T. Ngan, E. Janssens, P. Claes, A. Fielicke, M. T. Nguyen and P. Lievens, Phys. Chem. Chem. Phys., 17, 17584-17591, 2015.

[19] A. Shayeghi, R. L. Johnston, D. M. Rayner, R. Schfer and A. Fielicke, Angew. Chem. Int. Ed., 54, 10675 -10680, 2015.

[20] A. Shayeghi, R. Schäfer, D. M. Rayner, R. L. Johnston and A. Fielicke, J. Chem. Phys., 143, 024310, 2015.

[21] P. Ferrari, G.-L. Hou, O. V. Lushchikova, F. Calvo, J. M. Bakker and E. Janssens, Phys. Chem. Chem. Phys., 22, 11572-11577, 2020.

[22] J. van der Tol, D. Jia, Y. Li, V. Chernyy, J. M. Bakker, M. T. Nguyen, P. Lievens and E. Janssens, Phys. Chem. Chem. Phys., 19, 19360-19368, 2017.

[23] O. V. Lushchikova, D. M. M. Huitema, P. López-Tarifa, L. Visscher, Z. Jamshidi and J. M. Bakker, J. Phys. Chem. Lett., 10, 2151-2155, 2019.

[24] L. M. Ghiringhelli and S. V. Levchenko, Inorg. Chem. Commun., 55, 153-156, 2015.

[25] B. R. Goldsmith, J. Florian, J.-X. Liu, P. Gruene, J. T. Lyon, D. M. Rayner, A. Fielicke, M. Scheffler and L. M. Ghiringhelli, Phys. Rev. Materials, 3, 016002, 2019.

[26] P. Gruene, D. M. Rayner, B. Redlich, A. F. G. van der Meer, J. T. Lyon, G. Meijer and A. Fielicke, Science, 321, 674-676, 2008.

[27] L. M. Ghiringhelli, P. Gruene, J. T. Lyon, D. M. Rayner, G. Meijer, A. Fielicke and M. Scheffler, New J. Phys., 15, 083003, 2013.

[28] L. A. Mancera and D. M. Benoit, Phys. Chem. Chem. Phys., $15,1929,2013$

[29] S. Pan, R. Saha, S. Mandal and P. K. Chattaraj, Phys. Chem. Chem. Phys., 18, 11661-11676, 2016.
[30] Z. Jamshidi, K. Eskandari and S. M. Azami, Int. J. Quantum Chem., 113, 1981-1991, 2013.

[31] S. J. Grabowski, J. M. Ugalde, D. M. Andrada and G. Frenking, Chem. Eur. J., 22, $11317-11328,2016$.

[32] T. Zeng and M. Klobukowski, J. Phys. Chem. A, 112, 5236$5242,2008$.

[33] A. Yousef, S. Shrestha, L. A. Viehland, E. P. Lee, B. R. Gray, V. L. Ayles, T. G. Wright and W. H. Breckenridge, J. Chem. Phys., 127, 154309, 2007.

[34] S. M. Lang, P. Claes, N. T. Cuong, M. T. Nguyen, P. Lievens and E. Janssens, J. Chem. Phys., 135, 224305, 2011.

[35] P. Ferrari, J. Vanbuel, Y. L. T. Li, E. Janssens and P. Lievens, "Modifications of gas aggregation sources: The double laser ablation source approach," in Gas aggregation synthesis of nanoparticles, Weinheim, Wiley-VCH, 2017, pp. 57-78.

[36] A. Fielicke, A. Kirilyuk, C. Ratsch, J. Behler, M. Scheffler, G. von Helden and G. Meijer, Phys. Rev. Lett., 93, 023401, 2004.

[37] J. M. Bakker, V. J. F. Lapoutre, B. Redlich, J. Oomens, B. G. Sartakov, A. Fielicke, G. von Helden, G. Meijer and A. F. G. van der Meer, J. Chem. Phys., 132, 074305, 2010.

[38] M. Valiev, E. J. Bylaska, N. Govind, K. Kowalski, T. P. Straatsma, H. J. J. Van Dam, D. Wang, J. Nieplocha, E. Apra, T. L. Windus and W. A. de Jong, Comput. Phys. Commun., 1181, 1477, 2010.

[39] J. P. Perdew, A. Ruzsinszky and J. Tao, J. Chem. Phys., 123, 062201, 2005.

[40] J. P. Perdew, K. Burke and M. Ernzerhof, Phys. Rev. Lett., 77, 3865, 1996.

[41] J. Tao, J. P. Perdew, V. Staroverov, N. and G. E. Scuseria, Phys. Rev. Lett., 91, 146401, 2003.

[42] A. D. Becke, J. Chem. Phys., 98, 5648, 1993.

[43] O. A. Vydrov and G. E. Scuseria, J. Chem. Phys., 125, 234109, 2006.

[44] L. Goerigk and S. Grimme, WIREs Comput. Mol. Sci., 4, 576-600, 2014.

[45] F. Weigend and R. Ahlrichs, Phys. Chem. Chem. Phys., 7, 3297-3305, 2005.

[46] S. Grimme, S. Ehrlich and L. Goerigk, J. Comput. Chem., 32, 1456-1465, 2011.

[47] T. Lu and F. Chen, J. Comput. Chem, 33, 580-592, 2012.

[48] N. Mardirossian and M. Head-Gordon, Mol. Phys., 115, 2315-2372, 2017

[49] F. Neese, Rev.: Comput. Mol. Sci., 2, 73, 2012.

[50] Z. Jamshidi, O. V. Lushchikova, J. M. Bakker and L. Visscher, J. Phys. Chem. A, 124, 9004-9010, 2020.

[51] S. Grimme, WIREs Comput. Mol. Sci., 1, 211-228, 2011.

[52] K. Raghavachari, Theor. Chem. Acc., 103, 361-363, 2000.

[53] X.-Y. Li, X. Cao and Y. Zhao, J. Phys. B: At., Mol. Opt. Phys., 42, 065106, 2009. 